\title{
Il centenario del pH (1909-2009) - Parte seconda. Ma era proprio necessario sostituire l'equazione di Henderson con quella di Henderson-Hasselbalch?
}

The $100^{\text {th }}$ anniversary of the invention of $\mathrm{pH}$ (1909-2009) - Part II. Was it really necessary to replace the Henderson equation with that of Henderson-Hasselbalch?

\section{Francesco Sgambato*, Sergio Prozzo, Ester Sgambato, Rosa Sgambato, Luca Milano}

UO Medicina Interna, Ospedale Fatebenefratelli, Benevento

Ricevuto il 19 luglio 2011; accettato il 2 settembre 2011

disponibile online il 17 novembre 2011

\section{KEYWORDS}

$\mathrm{pH}$;

History of $\mathrm{pH}$;

Centenary of $\mathrm{pH}$;

Acid-base balance;

Henderson equation;

Henderson-Hasselbalch equation;

Logarithms in medicine.

\begin{abstract}
Summary
Introduction: The year 2009 marked the centenary of the "invention" of the concept of pH by the Danish chemist-mathematician Søren Peder Lauritz Sørensen (1868-1939), who was working at the time in the chemistry laboratories of the Carlsberg Brewery in Copenhagen. The occasion provides an opportunity to re-examine a concept that is crucial for the understanding of human life-namely, acid-base balance. This article provides an overview of acid-base pathophysiology and the historical events that led from the simple equation of Henderson to the much more complex one developed by Hasselbalch.

Conclusions: The authors conclude that the issue of acid-base balance would be easier to understand, more exciting, and even more pleasant if it were taught without recourse to the infamously abstruse Henderson-Hasselbalch equation. Unquestionably, the whole rationale underlying the understanding and clinical application of this vital concept is already inherent in the simpler, more manageable formula of Henderson (without logs), which is both useful and sufficient for use in medical school curricula.

(c) 2011 Elsevier Srl. All rights reserved.
\end{abstract}

\footnotetext{
* Corrispondenza: Ospedale Fatebenefratelli, viale Principe di Napoli 14/A - 82100 Benevento.
}

E-mail: sgambatof@gmail.com (F. Sgambato). 


\section{Introduzione}

"Quando Dio creò il mondo, cominciò, presumibilmente, con l'idrogeno. Esso è il primo elemento della tavola periodica... In principio c'erano il caos e l'idrogeno, alla fine, tra gli altri risultati della creazione, rimasero. . . l'equilibrio acido-base e il medico spesso confuso" (J.R. Elkinton) [1].

"Life is a struggle, not against sin, not against the Money Power, not against malicious 'animal magnetism', but against hydrogen ions" ("La vita è una lotta, non contro il peccato, non contro il Potere del Denaro, non contro il magnetismo animale 'maligno', ma contro gli ioni idrogeno") (H.L. Mencken, 1919) [2].

Bastano queste due frasi, di epoche diverse, per esprimere alcuni concetti fondamentali della fisiologia e della patologia umana, cioè: 1) l'importanza dell'idrogeno nella vita dell'uomo e 2) la difficoltà ad avvicinarsi al tema dell'equilibrio acido-base, che ha turbato varie generazioni di studenti e di medici.

Lo studio degli equilibri (acido-base, idroelettrolitico e osmolare) deve per forza rappresentare un "fondamentale" nella cultura medica ed è uno degli argomenti trasversali e unificanti nella formazione generale in Medicina, che sempre più è orientata alla cura delle patologie gravi nei pazienti critici, pluripatologici e, come tali, complessi, per cui meritevoli di una visione olistica, allargata a 360 gradi.

Nella nostra attività lavorativa quotidiana di medici (ospedalieri e non), nulla di quello che facciamo routinariamente non incide o non interferisce su queste materie e tale considerazione fa sì che questi temi siano argomenti di cui dobbiamo occuparci per forza. Essi sono praticamente ineludibili, a meno che non vogliamo autoingannarci o autoilluderci. Proprio i pazienti complessi sono più meritevoli di una cultura attenta a queste problematiche "sistemiche", che in definitiva rappresentano il nocciolo della visione olistica e della mentalità tipicamente internistica.

L'"ambiente interno" (milieu interieur) fu un'idea rivoluzionaria di Claude Bernard (1865) [3] e da essa sono originati il concetto di "equilibrio" elaborato da Lawrence J. Henderson nel 1908 [4-6], poi quello delle "interrelazioni fra gli equilibri biologici" espresso da Jacques Loeb nel 1916

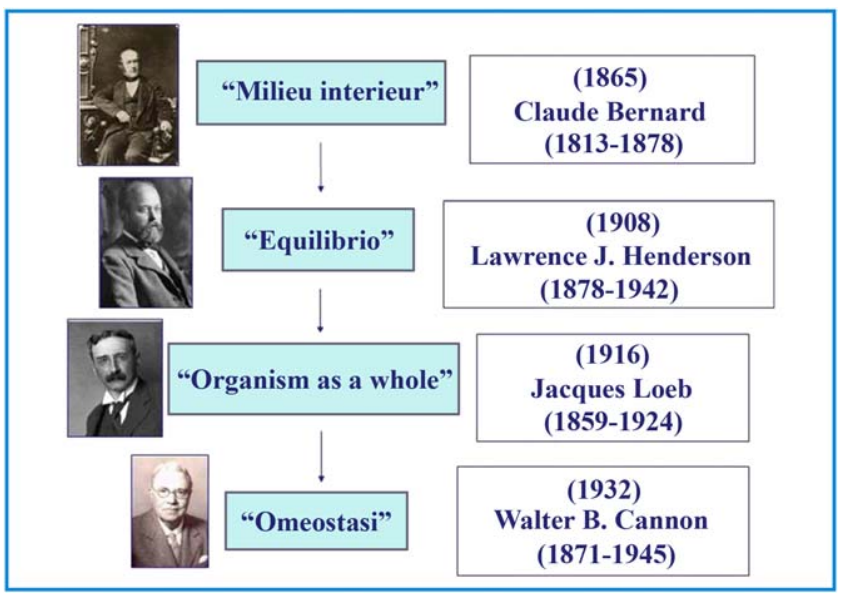

Figura 1 Le tappe storiche del concetto di equilibrio.

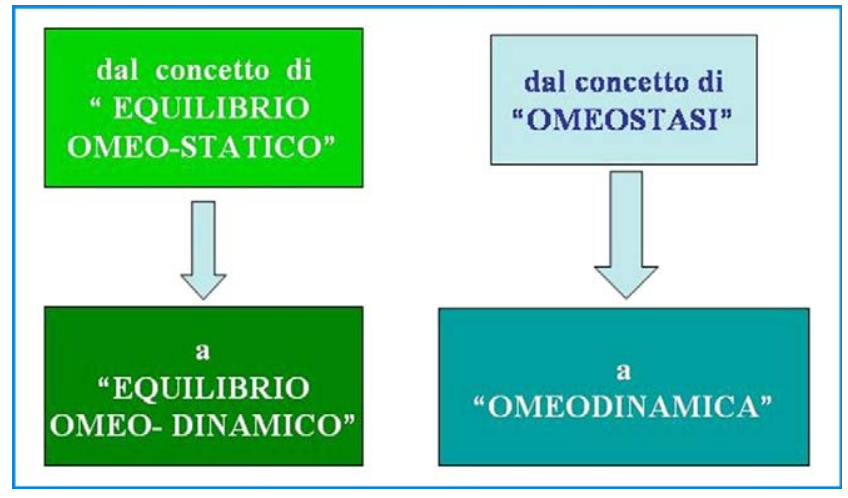

Figura 2 Dall"'omeostasi" di Cannon all" omeodinamica" attuale.

[7-9] e, alfine, quello di "omeostasi" coniato da Walter B. Cannon nel 1932 [10] ( fig. 1).

A proposito di "omeostasi" forse è opportuno perfezionare il pensiero di Cannon, riflettendo sul fatto che in natura non c'è nulla di statico, ma vi è un" "oscillazione permanente tra equilibrio e instabilità (disequilibrio)", per cui a buona ragione può essere necessario compiere un'ulteriore evoluzione dal concetto di equilibrio omeostatico di Cannon a quello di equilibrio omeodinamico. In altre parole, poiché la "stasi" non è una prerogativa stabile della condizione e della natura umana, è opportuno passare dal concetto di omeostasi a quello di omeodinamica ( fig. 2).

L'approccio a queste tematiche è indispensabile per il medico e il presente lavoro si prefigge di:

- far odiare meno l'equilibrio acido-base;

- far avvicinare al tema con più simpatia e con occhio clinico pratico;

- convincere che l'argomento può essere studiato facilmente, senza passare sotto le "forche caudine" dell'equazione di Henderson-Hasselbalch;

- far applicare l'equazione di Henderson nella pratica clinica (senza Hasselbalch) e convincere che, in tal modo, non si perde nulla in termini di scientificità.

\section{Premesse di fisiopatologia classica}

L'organismo umano, per effetto del suo metabolismo, produce in continuazione acidi che, secondo la definizione di Brønsted, sono sostanze capaci di generare idrogenioni (mentre le basi sono capaci di assorbire idrogenioni).

I vari metabolismi (protidico, glicidico e lipidico), attraverso le diverse tappe metaboliche, producono, come traguardo finale, tre sostanze: ammoniaca $\left(\mathrm{NH}_{3}\right)$, acqua $\left(\mathrm{H}_{2} \mathrm{O}\right)$ e anidride carbonica $\left(\mathrm{CO}_{2}\right)$, oltre a generare energia (ATP - adenosintrifosfato).

Ogni giorno, dai nostri processi metabolici vengono ricavati $15.000-20.000 \mathrm{mEq}$ (milliequivalenti) di $\mathrm{CO}_{2}$, che vengono poi escreti dai polmoni. Inoltre, una normale dieta provoca la formazione di $50-100 \mathrm{mEq} \mathrm{di} \mathrm{H}^{+}$nelle 24 ore. Basti pensare, semplicemente, che l'acqua potabile, erogata dal rubinetto, non ha $\mathrm{pH} 7,40$, bensì $\mathrm{pH}$ variabile intorno a 5 (solo l'acqua pura ha $\mathrm{pH} 7$ ); il latte ha $\mathrm{pH} \mathrm{6,6;} \mathrm{le} \mathrm{bibite} \mathrm{alla}$ cola hanno $\mathrm{pH} 2,8$; la soluzione fisiologica ha $\mathrm{pH}$ inferiore 
a 7,0 (e, quindi, non fisiologico); la soluzione glucosata ha pH oscillante, ma sempre acido, intorno a 4,5 o 5,0.

La maggiore preoccupazione del corpo umano, quindi, è quella di difendersi dall'acidità, perché esso normalmente produce idrogenioni $\left(\mathrm{H}^{+}\right.$, acidi) e non ossidrilioni $\left(\mathrm{OH}^{-}\right.$, basi), pertanto l'acidosi è molto più frequente dell'alcalosi.

La produzione di idrogenioni avviene in condizioni normali (fisiologiche), mentre l'alcalinità si crea solo in una situazione di patologia; non bisogna dimenticare, al riguardo, che l'idrogeno è l'atomo presente in maggiore abbondanza nelle cellule e che la maggiore quota dell'idrogeno è sotto forma di acqua.

Gli acidi prodotti normalmente, però, prima di raggiungere gli organi emuntori, passano nel sangue e tale passaggio, dalla produzione all'eliminazione, deve avvenire senza modificare la normale concentrazione idrogenionica.

Questa è la più sorprendente qualità dei nostri sistemi omeostatici, come espresso da L.J. Henderson già nel 1908: "The most significant and the most conspicious property of blood is the extraordinary ability to neutralize large amounts of acids or bases without losing its neutral reaction" ("La più importante e la più evidente proprietà del sangue è la sua straordinaria capacità di neutralizzare grandi quantità di acidi o basi senza perdere la sua reazione neutra") [4].

Sørensen, poi, nel 1909 dimostrò che: "La normale concentrazione dello ione idrogeno è un fattore essenziale in tutti i processi biologici" [11]. Tale concentrazione degli idrogenioni deve oscillare, di norma, intorno a $40 \mathrm{nEq} / \mathrm{L}$ (corrispondenti a un $\mathrm{pH}$ di 7,40 ) e variazioni di questi numeri possono alterare tutti $\mathrm{i}$ processi metabolici. Se ne può dedurre che gli ioni $\mathrm{H}^{+}$liberi in effetti sono presenti in concentrazioni estremamente basse (40 nanoequivalenti) e la loro stabilità in un range ristretto (da 16 a $160 \mathrm{nEq}$ ) è cruciale per il mantenimento della vita.

Se esprimiamo il loro valore normale con l'unità di misura dei grammiequivalenti per litro, si ottiene il valore di 0,00000004 , con sette zeri dopo la virgola seguiti dal numero 4 (il che corrisponde a un numero esponenziale negativo di $1,0 \times 10^{-7,4}$ e, cioè, a un pH di 7,40) [53].

Le difese poste in essere dall'organismo contro le possibili variazioni di questa concentrazione idrogenionica normale si basano su tre differenti sistemi (i tamponi, i polmoni e i reni) con diversi tempi di intervento.

I sistemi tampone corporei entrano in azione nel giro di pochi secondi e il sistema tampone bicarbonato/acido carbonico è il più importante di tutti, per tre motivi fondamentali:

- è presente in quantità maggiori rispetto agli altri $(65 \%$ del totale);

- è il più ubiquitario (è presente nel fluido interstiziale, nel plasma, negli eritrociti, nelle cellule, nelle ossa);

- è l'unico sistema che ha due vie di sfogo: una, rapida, attraverso i polmoni trasformandosi in $\mathrm{CO}_{2}$ (che viene eliminata nell'arco di pochi minuti) e l'altra ritrasformandosi in bicarbonato e idrogenioni, i quali ultimi vengono eliminati con il rene [12-14] ( fig. 3).

Il fondamentale segreto di questo sistema tampone è che l'acido carbonico è l'unico (fra tutti i sistemi tampone) ad avere una scappatoia all'esterno trasformandosi in qualchecosa di volatile $\left(\mathrm{CO}_{2}\right)$ più acqua residua, che viene eliminata.

Il rene collabora bene, però entra in azione più lentamente e impiega almeno 8-12 ore solo per dare inizio alla sua

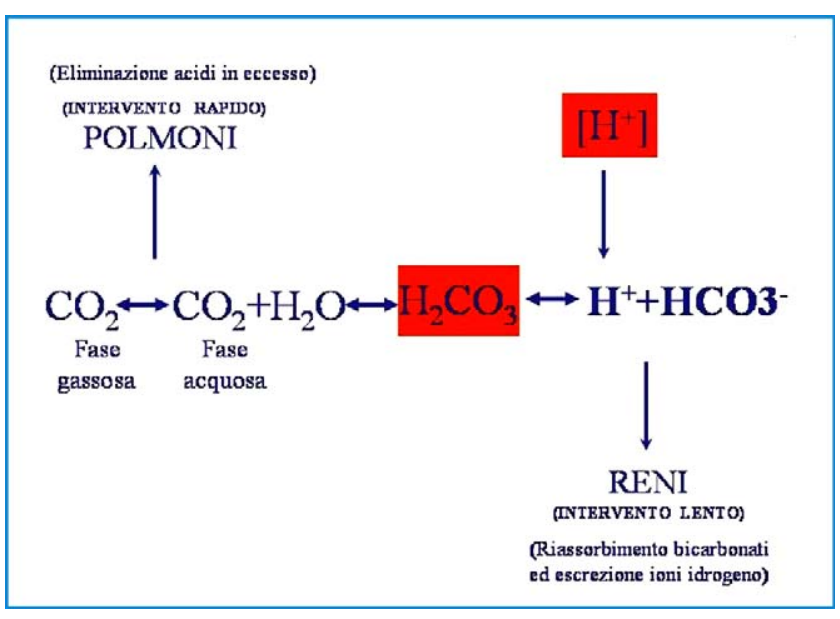

Figura 3 Lo schema fondamentale dell'equilibrio acido-base.

attività di correzione, che consiste, essenzialmente, nell'eliminare nelle urine gli acidi fissi e gli idrogenioni sotto forma di acqua o di ammonio o di fosfati, oltre all'indispensabile funzione di recuperare $i$ bicarbonati mediante il loro riassorbimento già nel tubulo prossimale. Circa il $90 \%$ dei bicarbonati viene riassorbito nel primo passaggio glomerulo-tubulare.

I sistemi tampone corporei, infatti, sono presenti per un totale di $2.400 \mathrm{mEq}$ (globalmente), mentre gli acidi da tamponare prodotti nelle 24 ore (volatili e non volatili) ammontano a 15.000-20.000 mEq. Da questa discrepanza, tra gli acidi prodotti nelle 24 ore $(15.000-20.000 \mathrm{mEq})$ e il totale dei sistemi tampone corporei, si intuisce la necessità che i tamponi siano continuamente rinnovati nel corso della giornata, pena l'esaurimento delle scorte disponibili. A tale funzione è deputato il rene con la sua attività di rigenerazione e riassorbimento dei bicarbonati, oltre a quella di eliminazione degli acidi fissi.

Ai tamponi organici (basi) è affidato, invece, il ruolo di emergenza e istantaneità dell'intervento, mentre al polmone spetta il compito di eliminare gli acidi volatili $\left(\mathrm{CO}_{2}\right)$ ( fig. 4).

\section{Difese contro le variazioni della $\left[\mathrm{H}^{+}\right]$}

Tempo di azione

1) Sistemi tampone acido-base Frazioni di secondo

2) Polmoni: Ventilazione

1-15 minuti

3) Reni: eliminazione di urina

Ore - giorni

acida (o alcalina) e recupero

dei bicarbonati

Figura 4 Tempi di intervento dei meccanismi di difesa. 
Se ci fossero solo i bicarbonati potremmo sopravvivere alcune ore (mezza giornata circa); se ci fossero solo i polmoni potremmo resistere qualche giorno mediante l'iperventilazione, fino a quando la fatica muscolare ce lo consentisse; se ci fossero solo i reni, mentre essi arrivano a funzionare a pieno regime saremmo già morti per eccesso di acidi non più tamponabili.

\section{La fondamentale (ma poco famosa) equazione di Henderson}

Laurence J. Henderson (1878-1942), professore di Fisiologia ad Harvard (Stati Uniti) ( fig. 5), abituato a studiare i sistemi biologici dal punto di vista matematico e fisico-chimico e affascinato dalla proprietà del sangue di tamponare grandi quantità di acidi e basi, descrisse i sistemi tampone, li caratterizzò matematicamente ed elaborò (1908) la sua geniale e fondamentale formula, conosciuta come "equazione di Henderson" ( fig. 6) [4].

In questa formula già era stata sintetizzata benissimo tutta la problematica dell'equilibrio acido-base, ovverosia che la concentrazione degli idrogenioni (e quindi l'acidità o la basicità di una soluzione) è data dal rapporto quantitativo esistente fra la concentrazione dell'acido carbonico $\left(\mathrm{H}_{2} \mathrm{CO}_{3}\right.$, al numeratore) e la concentrazione dei bicarbonati $\left(\mathrm{HCO}_{3}{ }^{-}\right.$, al denominatore) secondo una costante $\mathrm{K}$.

L'acido carbonico $\left(\mathrm{H}_{2} \mathrm{CO}_{3}\right)$, poi, non è altro che $\mathrm{CO}_{2}$ disciolta in acqua $\left(\mathrm{CO}_{2}+\mathrm{H}_{2} \mathrm{O}=\mathrm{H}_{2} \mathrm{CO}_{3}\right)$ e presenta una relazione perfettamente lineare con la pressione dell'anidride carbonica $\left(\mathrm{pCO}_{2}\right)$, cioè "più aumenta la pressione della $\mathrm{CO}_{2}$, più quest'ultima si discioglie nell'acqua stessa e forma acido carbonico".

Considerato che la misurazione dell'acido carbonico presenta difficoltà tecniche, mentre la misurazione della $\mathrm{pCO}_{2}$ è più semplice, per questi motivi l'equazione di Henderson venne espressa anche in un altro modo, sostituendo, al numeratore, l'acido carbonico con la $\mathrm{pCO}_{2}$. Tale variazione

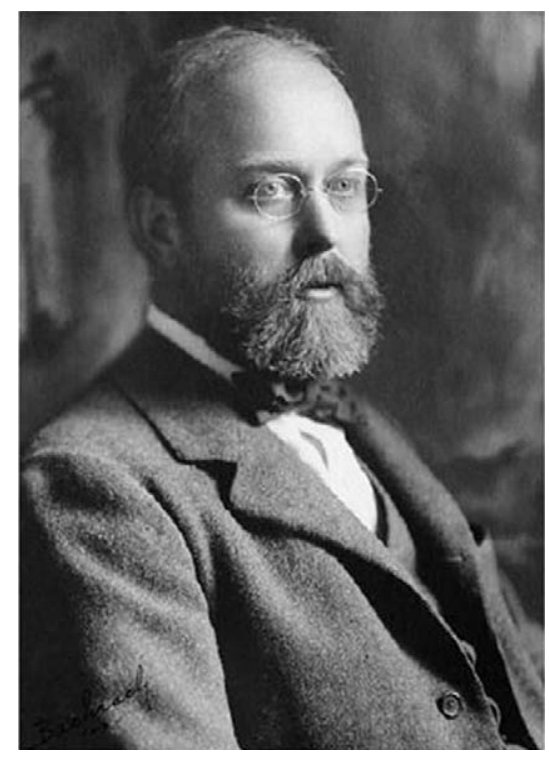

Figura 5 Laurence J. Henderson (1878-1942), professore di Fisiologia ad Harvard (Stati Uniti).

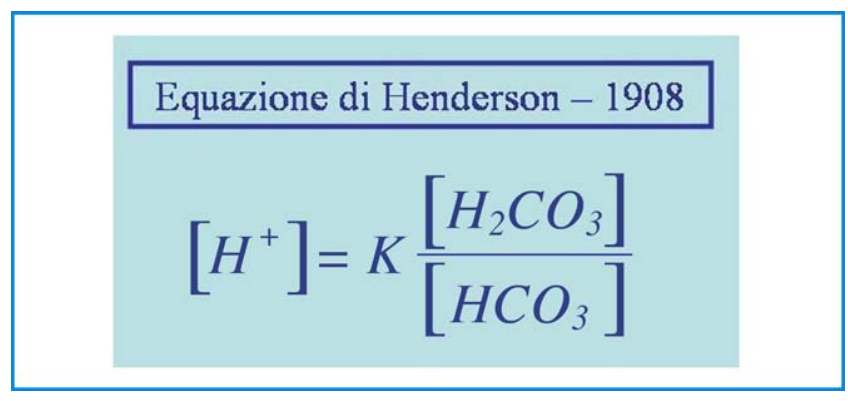

Figura 6 L'equazione di Henderson.

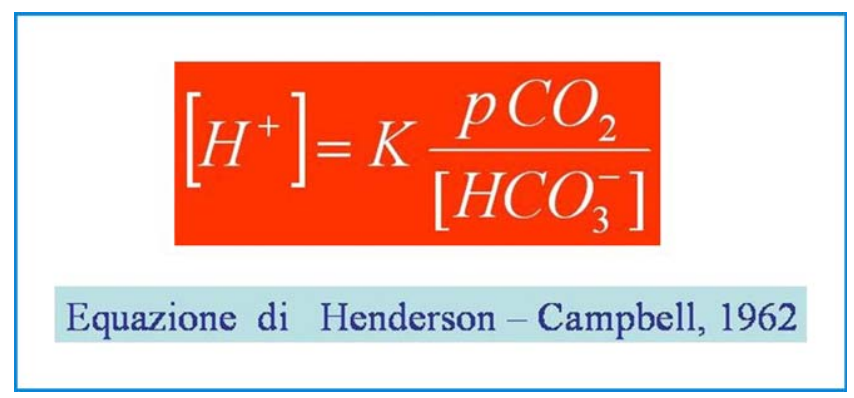

Figura 7 L'equazione di Henderson-Campbell (1962).

fu proposta da E.J.M. Campbell nel 1962 [15], dando origine alla definitiva equazione di Henderson-Campbell che è la più semplice e la più pratica da utilizzare ( $f$ ig. 7).

Essa, infatti, ci consente di passare dalla chimica (spesso antipatica) alla clinica (a noi più congeniale). Considerato, infatti, che a regolare la $\mathrm{pCO}_{2}$ è deputato prevalentemente il polmone e a regolare la concentrazione del bicarbonato è deputato prevalentemente il rene, possiamo concludere che la concentrazione degli idrogenioni è la risultante del rapporto tra la funzione del polmone e la funzione del rene (equazione umanizzata) ( fig. 8). Questa immagine semplifica la memorizzazione della formula ed è semplice richiamarla alla mente perchè il polmone sta al di sopra del diaframma (linea della frazione) e il rene sta al di sotto di esso.

In condizioni patologiche questo equilibrio si può rompere e si hanno le alterazioni acido-base, isolate o miste. Se aumenta la $\mathrm{pCO}_{2}$ (per esempio in caso di una patologia polmonare: cuore polmonare, polmonite, asma, broncopneumopatia cronica ostruttiva ecc.) si avrà un aumento degli idrogenioni e, quindi, un'acidosi di origine respiratoria; se invece diminuisce la $\mathrm{pCO}_{2}$ (per esempio a causa di un'iperventilazione in corso di crisi di ansia) diminuiscono gli idrogenioni e si avrà un'alcalosi respiratoria.

Se diminuiscono i bicarbonati (al denominatore) si avrà un aumento degli idrogenioni liberi e, di conseguenza, un'acidosi di origine metabolica; se aumentano i bicarbonati, diminuiscono gli idrogenioni liberi e si avrà una alcalosi metabolica.

Queste sono le classiche condizioni che etichettano i quattro disturbi "semplici" dell'equilibrio acido-base (acidosi respiratoria, acidosi metabolica, alcalosi respiratoria, alcalosi metabolica) e non ci vuole molto a individuarli bene.

Più complesse sono la comprensione e la correzione dei disturbi "misti", ma ugualmente essi sono più facilmente 


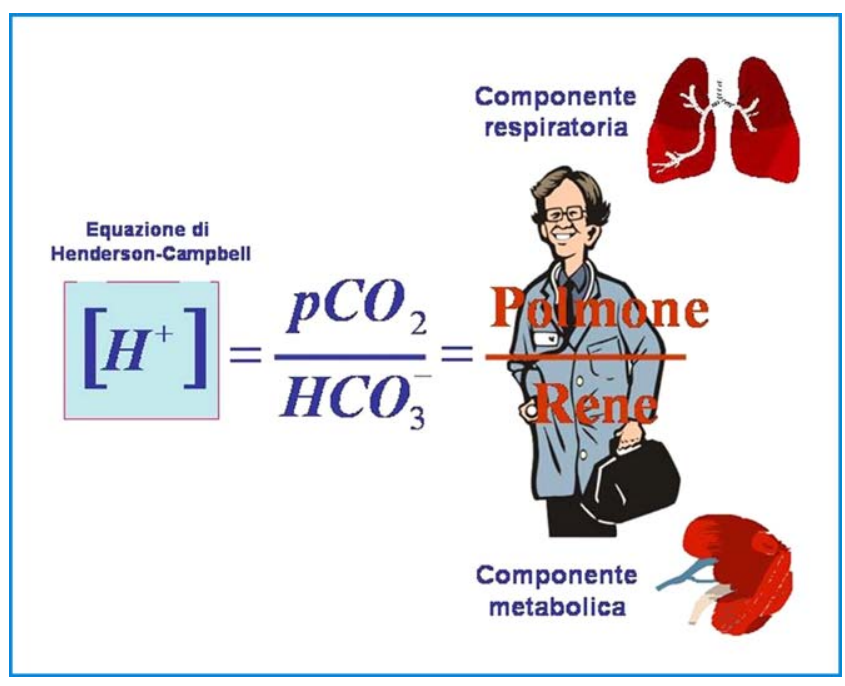

Figura 8 La concentrazione degli idrogenioni è la risultante del rapporto tra la funzione polmonare e quella renale.

inquadrabili se si fa riferimento alla formula di HendersonCampbell.

Dall'equazione di Henderson, generalmente poco nota, fu elaborata la famigerata equazione di HendersonHasselbalch, molto nota, in cui comparvero gli antipatici logaritmi (fig. 9).

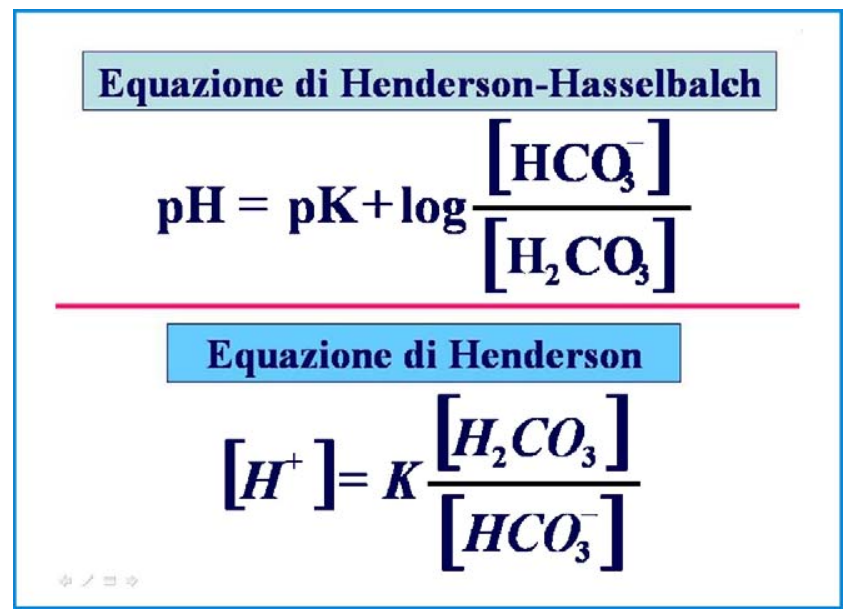

Figura 9 Le due equazioni poste a confronto.

\section{Il concetto dei compensi attesi (o "adattamenti" previsti)}

Quando il disturbo primario è "respiratorio", scatta il meccanismo di compenso metabolico del rene, che interviene regolando l'escrezione di ioni idrogeno e aumentando o diminuendo il riassorbimento dei bicarbonati. Quando il disturbo primario è "metabolico" scatta il meccanismo di compenso respiratorio dei polmoni che regolano la fuoriuscita di $\mathrm{CO}_{2}$.

La figura 10 illustra il sistema acido-base come una bilancia in equilibrio, ma il senso non cambia: miscelando $\mathrm{pCO}_{2}$

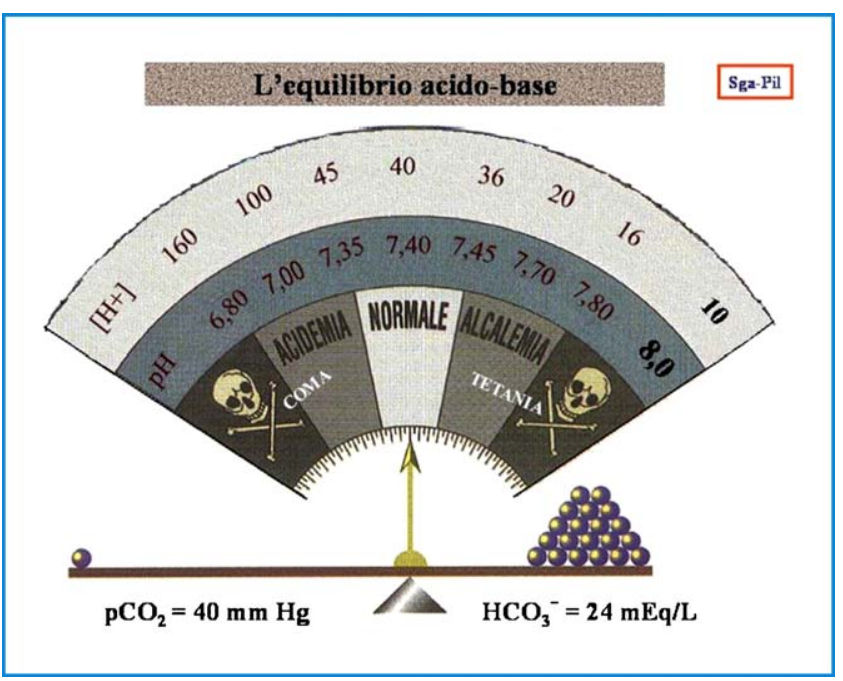

Figura 10 La bilancia dell'equilibrio acido-base.

(1 parte) e 20 parti di bicarbonato si ottiene la condizione normale, con la concentrazione idrogenionica uguale a $40 \mathrm{nEq} / \mathrm{L}$ (e il pH uguale a 7,40).

Come afferma Luciano de Crescenzo: "L'equilibrio deve essere inteso in questo modo: la doccia ha due manopole, una per l'acqua calda e una per l'acqua fredda. Manovrando, ora l'una ora l'altra, è possibile ottenere la giusta temperatura" [16].

Miscelando $\mathrm{CO}_{2}$ e bicarbonati in maniera equilibrata sui due piatti della bilancia si ottiene una concentrazione normale di idrogenioni e si preserva la buona sopravvivenza.

Si può avere la giusta temperatura dell'acqua o miscelando poca acqua calda con poca acqua fredda, oppure molta acqua calda con molta acqua fredda; in questo secondo caso, ovviamente, si avrà un dispendio maggiore di energia.

La natura umana dispone di un programma matematico di miscelazione automatica, migliore dei migliori miscelatori esistenti in commercio, e in fisiologia umana le risposte compensatorie (cioè gli "adattamenti" per miscelare al punto giusto) sono stati studiati con grande attenzione e vengono definiti compensi naturalmente "attesi" (o "adattamenti" previsti) dall'organismo umano. Essi sono espressi nella figura 11.

A ogni "disturbo primario" del sistema acido-base conseguono "adattamenti fisiologici", già calcolabili, in buona approssimazione matematica, fino a certi limiti naturali oltre i quali non si va.

Questo schema sintetico è cruciale e conviene tenerlo, plastificato, nel taschino del camice per l'utilizzo veloce nelle situazioni cliniche critiche.

Le figure 3, 8, 11 riassumono in maniera chiara tutti $i$ concetti meritevoli di essere memorizzati che, quindi, rappresentano " $i$ fondamentali" in questo campo, tali da meritarsi il titolo di "diapositive da salvare nell'incendio della biblioteca" e bastevoli per la comprensione del tema acidobase, cosiddetto "ostico" finora.

Come si può constatare, non sono presenti logaritmi, a dimostrazione ulteriore che essi non sono indispensabili per apprendere questo delicato e vitale argomento. 


\begin{tabular}{|c|c|c|c|}
\hline \multicolumn{4}{|c|}{ COMPENSO RENALE E RESPIRATORIO AI DISTURBI PRIMARI DELL'EQUILIBRIO ACIDO-BASE NELL'UOMO } \\
\hline DISORDINE & $\begin{array}{l}\text { ALTERAZIONE } \\
\text { PRIMITIVA } \\
\end{array}$ & RISPOSTA COMPENSATORIA PREVISTA & LIMTI \\
\hline $\begin{array}{l}\text { Acidosi } \\
\text { metabolica }\end{array}$ & $\downarrow\left[\mathrm{HCO}_{3}^{-}\right]$ & $\begin{array}{c}\text { Diminuzione di } 1,2 \mathrm{mmHg} \text { della } \mathrm{pCO}_{2} \text { per ogni } \\
\mathrm{mEq} / \mathrm{l} \text { di caduta dei bicarbonati }\left[\mathrm{HCO}_{3}^{-}\right]\end{array}$ & $10-15 \mathrm{mmHg}$ \\
\hline $\begin{array}{l}\text { Alcalosi } \\
\text { metabolica }\end{array}$ & $\uparrow\left[\mathrm{HCO}_{3}\right]$ & $\begin{array}{l}\text { Aumento di } 0,7 \mathrm{mmHg} \text { della } \mathrm{pCO}_{2} \text { per ogni } \\
\mathrm{mEq} / \mathrm{l} \text { di aumento dei bicarbonati }\left[\mathrm{HCO}_{3}^{-}\right]\end{array}$ & $55 \mathrm{mmHg}$ \\
\hline $\begin{array}{l}\text { Acidosi } \\
\text { respiratoria }\end{array}$ & $\uparrow \mathrm{pCO}_{2}$ & & \\
\hline - Acuta & & $\begin{array}{l}\text { Aumento di } 1 \mathrm{mEq} / \mathrm{l} \text { dei bicarbonati }\left[\mathrm{HCO}_{3}^{-}\right] \\
\text {per ogni } 10 \mathrm{mmHg} \text { di aumento della } \mathrm{pCO}_{2}\end{array}$ & $30 \mathrm{mEq} / \mathrm{l}$ \\
\hline - Cronica & & $\begin{array}{l}\text { Aumento di } 3,5 \mathrm{mEq} / \mathrm{l} \text { dei bicarbonati }\left[\mathrm{HCO}_{3}^{-}\right] \\
\text {per ogni } 10 \mathrm{mmHg} \text { di aumento della } \mathrm{pCO}_{2}\end{array}$ & $45 \mathrm{mEq} / \mathrm{l}$ \\
\hline $\begin{array}{l}\text { Alcalosi } \\
\text { respiratoria }\end{array}$ & $\downarrow \mathrm{pCO}_{2}$ & & \\
\hline - Acuta & & $\begin{array}{l}\text { Riduzione di } 2 \mathrm{mEq} / \mathrm{l} \text { dei bicarbonati }\left[\mathrm{HCO}_{3}^{-}\right] \\
\quad \text { per ogni } 10 \mathrm{mmHg} \text { di diminuzione della } \mathrm{pCO}_{2}\end{array}$ & $18 \mathrm{mEq} / \mathrm{l}$ \\
\hline - Cronica & & $\begin{array}{l}\text { Riduzione di } 5 \mathrm{mEq} / \mathrm{l} \text { dei bicarbonati }\left[\mathrm{HCO}_{3}^{-}\right] \\
\quad \text { per ogni } 10 \mathrm{mmHg} \text { di diminuzione della } \mathrm{pCO}_{2}\end{array}$ & $12-15 \mathrm{mEq} / \mathrm{l}$ \\
\hline
\end{tabular}

Figura 11 Tabella dei compensi "attesi" (o "adattamenti previsti").

\section{Dalla "semplicità" di Henderson alla "complessità" di Hasselbalch}

Nel1908 (con Henderson) tutto era facilmente comprensibile e la sua equazione, che non presentava i logaritmi, già esprimeva benissimo tutti i rapporti esistenti all'interno del complesso sistema acido-base.

Poi Niels Bjerrum (1879-1958) ( fig. 12), professore universitario di Veterinaria e Agricoltura, introdusse i logaritmi in questa equazione e Karl Albert Hasselbalch (1874-1962) ( fig. 12) vi apportò, nel 1916, il concetto di pK, dal che venne trasferita in Chimica, in Biochimica e poi anche in Medicina
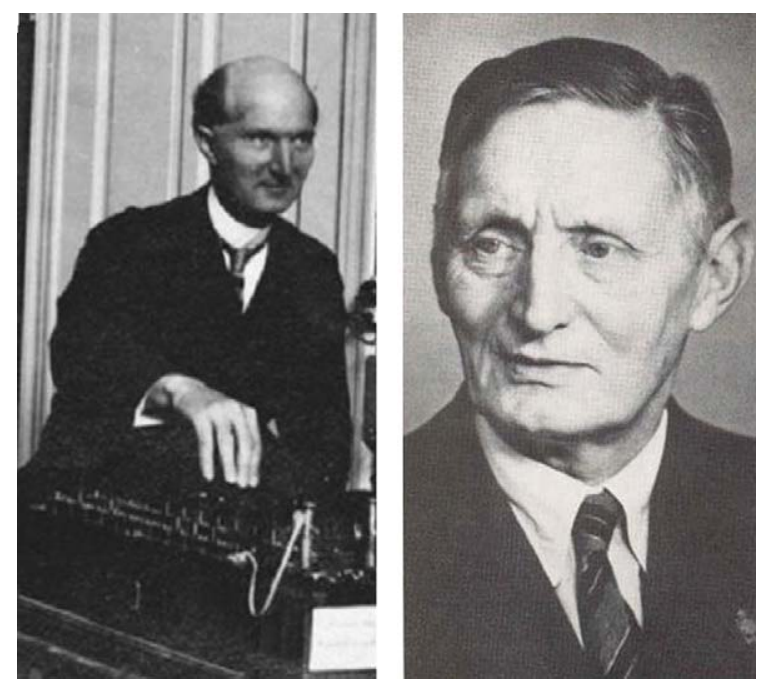

Figura 12 Da sinistra: Niels Bjerrum e Karl Albert Hasselbalch.
( fig. 9). Il pH (logaritmo negativo in base 10 della concentrazione degli idrogenioni) è dato dal pK (logaritmo negativo in base 10 della costante di dissociazione) più il logaritmo della concentrazione dei bicarbonati fratto il logaritmo della concentrazione dell'acido carbonico.

Si può affermare, quindi, che l'idea di trascriverla in formato logaritmico sia venuta, per primo, al chimico danese Bjerrum perché lo stesso Hasselbalch, nel suo lavoro del 1916, dichiara che, nello scrivere la nuova equazione, sta seguendo un "lavoro non pubblicato di Bjerrum" [17,18]. In tal modo egli procede a fondere la formula dei tamponi di Henderson con la scala del $\mathrm{pH}$ di Sørensen, diventando praticamente immortale e oscurando nei libri di testo non solo gli stessi Henderson e Sørensen, ma anche Guldberg e Waage [19].

Non bisogna mai dimenticare, infatti, che anche l'equazione di Henderson non è altro che una applicazione della fondamentale "legge di azione di massa", genialmente intuita da Guldberg e Waage nel 1864 [20] (più di quarant'anni prima) e perfezionata dagli stessi autori nel 1879 [21].

L'equazione formulata da Henderson è solo un" "applicazione" di questa legge generale, limitatamente alla miscela di un acido (acido carbonico) con la sua base coniugata (bicarbonato), ed estrapolata in seguito ai suoi studi sulle azioni dei tamponi nel 1908.

Si ebbe, poi, nel 1916, quella che nel mondo universitario veterinario e dell'agricoltura è ricordata come equazione di Bjerrum e in campo medico è diventata la famigerata equazione di Henderson-Hasselbalch.

È sufficiente mettere a confronto le due formule per rendersi conto della diversa complessità, eclatante già a prima vista ( fig. 9).

Da allora siamo stati indotti a seguire una via impervia, sicuramente non pratica e non facilmente percorribile 


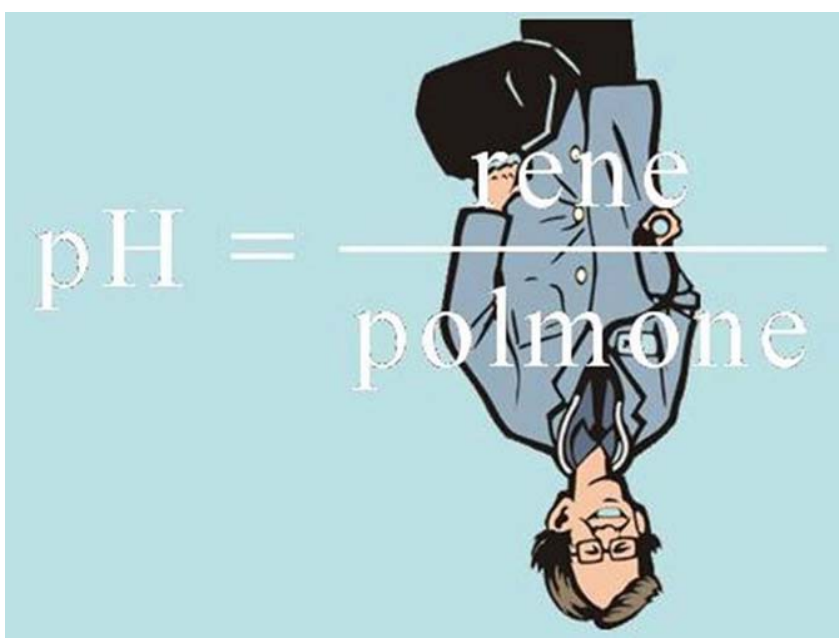

Figura 13 L'equazione "invertita".

da tutti e, come i ciechi di Bruegel (Museo di Capodimonte, Napoli), siamo caduti proprio nel fossato uno dietro l'altro.

Non si dice un'eresia se si attribuisce a questa formula (utilizzata normalmente, in ogni libro, quando si incomincia lo studio dell'argomento) la responsabilità del mancato entusiasmo di quanti si avvicinano a questo tema. Infatti conosciamo bene, tutti noi, la cordiale antipatia dei medici per le formule chimiche, quale retaggio che ci trasciniamo dietro dai tempi liceali.

Nella nostra scelta professionale giovanile, pensavamo di dover visitare i pazienti, auscultare i cuori, palpare i fegati, percuotere $i$ toraci, ovverosia di confrontarci con qualchecosa di "umano", ma non immaginavamo di dover avere a che fare con logaritmi, frazioni, formule chimiche ecc.

Lo studio dell'equilibrio acido-base può fare riferimento, invece, alla sola equazione di Henderson-Campbell ( fig. 8), che consente di capire subito il concetto chiave: più alta è la $\mathrm{pCO}_{2}$, più alta è la concentrazione idrogenionica, con un rapporto diretto e lineare.

Al contrario, con la formula di Hasselbalch bisogna ragionare all'inverso: se aumenta la $\mathrm{pCO}_{2}$ diminuisce il $\mathrm{pH}$, il che significa che aumentano gli idrogenioni (tutti equilibrismi superflui), perché non bastava il logaritmo ma doveva anche essere "logaritmo negativo" (fig. 13). Questo, a nostro giudizio, crea ulteriore fastidio e confusione, visto che il vero nemico da cui difendersi non è il $\mathrm{pH}$, ma sono gli idrogenioni.

Nell'organismo non ci sono i logaritmi della concentrazione degli idrogenioni, ma ci sono solo gli idrogenioni; né l'organismo sa calcolare $i$ logaritmi (come pure la maggior parte di noi medici).

D'altronde, gli elettrodi dell'emogasanalizzatore misurano l'attività idrogenionica, che solo in un secondo momento viene tramutata in $\mathrm{pH}$ con un ulteriore calcolo, che si potrebbe evitare. Per tali motivi ci siamo convinti che gli addetti all'equilibrio acido-base (come gli "equilibristi") siano stati abituati a stare con la testa in giù e i piedi per aria: praticamente vivono e ragionano, costantemente, in senso inverso. Basti pensare, tra l'altro, che chiamano "eccesso base" quello che in realtà è un "difetto di basi".

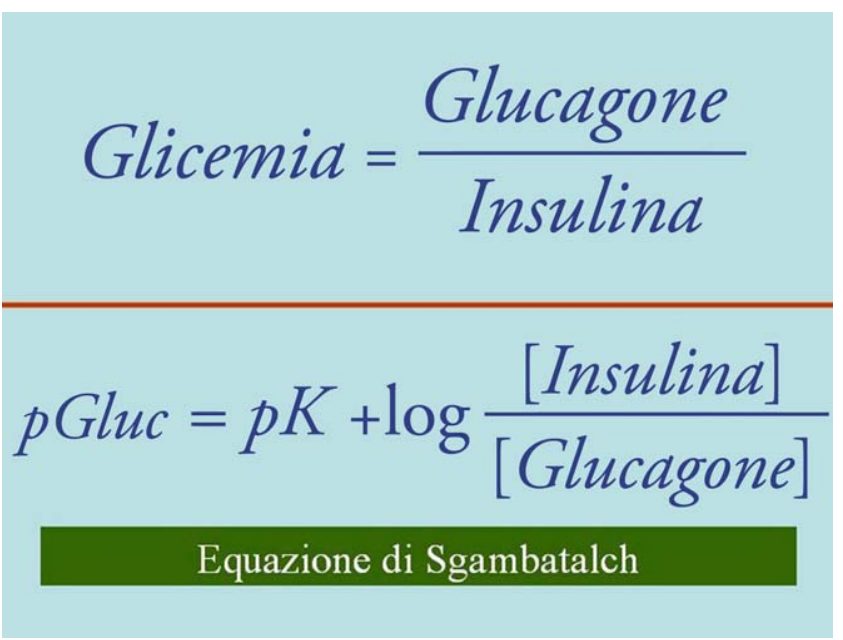

Figura 14 Dalla fisiologia semplice a quella "complicata".

In effetti tutta la terminologia utilizzata è ingombrante, come già scrivevano molti anni fa (1963) Schwartz e Narins: "Noi evitiamo l'uso dei termini 'bicarbonati standard' ed 'eccesso base' perché essi non aggiungono alcunché di valore pratico e semplicemente espandono il sempre crescente lessico dell'equilibrio acido-base" [22,23].

Possiamo immaginare che cosa sarebbe successo se, a proposito del diabete, tanto per fare un esempio, non ci fosse stato insegnato che la glicemia dipende dal rapporto fra l'azione del glucagone e quella dell'insulina (fig. 14), ma ci avessero detto che il pGluc (logaritmo negativo in base 10 della concentrazione del glucosio) è dato dal pK (logaritmo negativo in base 10 della costante di dissociazione) più il logaritmo della concentrazione dell'insulina fratto il logaritmo della concentrazione del glucagone (fig. 14).

Se qualcuno l'avesse, poi, chiamata equazione di Sgambatalch (nome altisonante e straniero), avremmo corso il rischio di ritrovarcela sui libri di testo e si può essere sicuri che nessuno di noi avrebbe studiato e capito anche il diabete.

In maniera analoga è facile intuire che nessuno si sarebbe mai sognato di trasformare il valore della potassiemia e/o della sodiemia utilizzando una forma logaritmica complicata, come avvenne per gli idrogenioni.

In definitiva, invece, l'equilibrio acido-base sarebbe rimasto un argomento semplice se studiato con l'equazione di Henderson senza i logaritmi e memorizzato con pochi schemi pratici (figg. 3, 8, 10), praticamente con un atteggiamento non da equilibristi ma da equilibrati.

\section{Qualche precisazione sui logaritmi}

Il logaritmo di un numero qualsiasi è la potenza a cui il numero base 10 deve essere elevato per eguagliare quel numero.

Per esempio, il logaritmo del numero 100 è 2: infatti, elevando il numero 10 alla potenza $2\left(1 \times 10^{2}\right)$ si ottiene 100 .

Il logaritmo di 10 è $1\left(1 \times 10^{1}=10\right)$.

Il logaritmo di 1 è, in qualsiasi base, uguale a 0 (zero). 


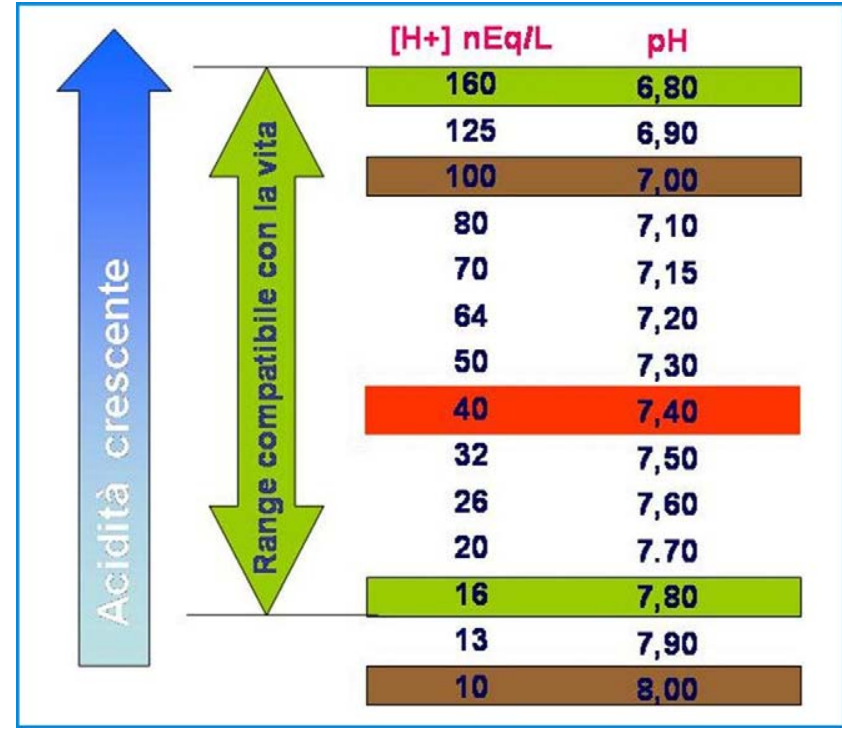

Figura 15 Rapporti fra concentrazioni idrogenioniche e scala del $\mathrm{pH}$.

Quando il numero in questione è inferiore a 1, il logaritmo corrispondente avrà segno negativo.

Il logaritmo di 0,1 è $-1\left(1 \times 10^{-1}=0,1\right)$.

Il logaritmo di 0,01 è $-2\left(1 \times 10^{-2}=0,01\right)$.

In conclusione, quindi, non vi è nulla di difficile. Tuttavia, visto che l'utilizzo dei logaritmi non è indispensabile in Medicina, perché rendere complicato ciò che è facile da apprendere senza superflue sovrapposizioni?

Un altro punto a sfavore dei logaritmi, poi, è anche di tipo tecnico, in quanto i logaritmi appiattiscono le differenze e portano a sottovalutare le variazioni rispetto alla norma.

Quando il pH, per esempio, cambia di sole 0,3 unità, apparentemente minime, da 7,4 a 7,1, la concentrazione idrogenionica già raddoppia, da 40 a $80 \mathrm{nEq} / \mathrm{L}$ raggiungendo livelli pericolosi per la vita ( fig. 15). Al contrario, il passaggio dal valore normale di $40 \mathrm{nEq} / \mathrm{L}$ ai successivi incrementi unitari $(41,42,43,44, \ldots 50, \ldots 60$ ecc.) rende più chiara la comprensione delle variazioni quantitative.

Di conseguenza, con l'utilizzo dei nanoequivalenti, le entità delle alterazioni della concentrazione idrogenionica sarebbero più facili da interpretare e farebbero scattare in anticipo i meccanismi d'allarme per la eventuale loro correzione.

\section{Qualche precisazione sul $\mathrm{pH}$ e sulle acrobazie da compiere per calcolarlo e interpretarlo}

Il pH è il "logaritmo negativo della concentrazione degli idrogenioni" $\left(\left[\mathrm{H}^{+}\right]\right.$, le parentesi quadre esprimono il concetto di "concentrazione"), cioè quella potenza a cui bisogna elevare il numero 10 per eguagliare la concentrazione degli idrogenioni misurati.

L'esponente della potenza che esprime la concentrazione degli ioni $\mathrm{H}^{+}$, cambiato di segno, diventa immediatamente il pH.
Quando la $\left[\mathrm{H}^{+}\right]$è 0,01 , cioè $10^{-2}$, allora il $\mathrm{pH}$ è pari a -2 , e se la $\left[\mathrm{H}^{+}\right]$è 0,0001 , cioè $10^{-4}$, allora il pH diventa -4 . Infatti, come abbiamo detto, tutti i logaritmi dei numeri più piccoli di 1 si esprimono con numeri negativi, mentre quelli dei numeri più grandi di 1 risultano in numeri positivi.

Il pH normale del sangue, che è il logaritmo di un numero inferiore a $1\left(0,00000004\right.$, ossia $\left.1,0 \times 10^{-7,4}\right)$, dovrebbe risultare negativo, ma Sørensen ritenne più conveniente esprimere la scala del $\mathrm{pH}$ con numeri positivi e, invertendo gli addendi, definì il $\mathrm{pH}$ come il logaritmo negativo della $\left[\mathrm{H}^{+}\right]$:

$$
\mathrm{pH}=-\log \left[\mathrm{H}^{+}\right]
$$

Il logaritmo normale di 0,00000004 è uguale a $-7,40$ (infatti 10 elevato a $-7,40$, cioè $10^{-7,4}$, è uguale a 0,00000004), ma Sørensen lo fece diventare positivo per praticità.

A causa di questo cambiamento "arbitrario" del segno numerico, da negativo a positivo, ne consegue che più è alta la concentrazione degli idrogenioni più è basso il $\mathrm{pH}$.

E questa è la spiegazione a uno dei principali motivi di confusione per gli studenti. Infatti, la loro domanda più classica è la seguente: "If $\mathrm{pH}$ is a measure of acidity, then how is it that pH goes up when the acidity goes down?" ("Se il $\mathrm{pH}$ è una misura dell'acidità, come fa il pH a salire quando l'acidità diminuisce?") [24].

A conferma di tali difficoltà di approccio all'argomento, Watters in un interessante studio sulla reale conoscenza del $\mathrm{pH}$ da parte degli studenti riportò, come sottotitolo del suo articolo, la risposta che gli aveva dato uno studente durante le sue interviste: "I don't know what the log actually is, I only know where the button is on my calculator" ("lo non so che cos'è il logaritmo, io so solo dov'è il tasto sulla mia calcolatrice") [25].

Importante, quindi, è procurarsi una buona calcolatrice che preveda anche il calcolo dei logaritmi, altrimenti essa non serve al nostro scopo nell'ambiente acido-base.

\section{Approfondimento sugli aspetti chimico- matematici: "quantità idrogenionica", "concentrazione idrogenionica", "attività degli ioni idrogeno"}

Precisiamo subito che, in questo ambito scientifico, molta confusione nasce da problemi di alta rilevanza in chimica pura, ma poco significativi in ambito medico-clinico pratico, cui vuole far riferimento il nostro lavoro.

In ogni caso è necessario e utile sapere che esistono differenze di significato fra il concetto di "quantità idrogenionica" e quello di "concentrazione idrogenionica", da non confondere, e ancor più con quello di "attività degli ioni idrogeno" (o "concentrazione idrogenionica realmente efficace").

L'idrogeno è formato da un nucleo (costituito da un neutrone e un protone con carica positiva) e da un elettrone, che ruota intorno a esso, come satellite carico negativamente. Quando l'atomo di idrogeno perde l'elettrone, il protone con carica positiva (o ione idrogeno) diventa "attivo" e genera l" "attività chimica". Esso diventa una sorta di chimico "agitatore" che suscita un elevato grado 
di reattività e instabilità tra i suoi "vicini di casa", ionici e molecolari [1].

L'acidità in una soluzione acquosa è dovuta proprio alla presenza degli idrogeno-ioni liberi $\left(\mathrm{H}^{+}\right)$.

Il grado di acidità è espresso in termini di "concentrazione" di $\mathrm{H}^{+}$, con il simbolo delle parentesi quadre $\left[\mathrm{H}^{+}\right]$, per significare le "moli per litro", e bisogna tener conto che, per quanto riguarda l'idrogeno, la concentrazione e la molarità hanno lo stesso valore matematico perché l'idrogeno ha peso atomico 1.

La mole (come utile promemoria per gli altri smemorati come noi) rappresenta per definizione "la quantità di particelle pari al peso molecolare della sostanza (espressa in grammi)".

La $\left[\mathrm{H}^{+}\right]$rappresenta, quindi, la concentrazione molare di ioni $\mathrm{H}^{+}$in una soluzione ed è una descrizione "quantitativa" della sua acidità espressa in moli.

Tuttavia, gli ioni idrogeno in una soluzione non sono protoni liberi di fluttuare naturalmente. Essi sono in realtà collegati alle altre molecole del solvente e, per questo motivo, gli ioni $\mathrm{H}^{+}$in acqua vengono spesso descritti come ioni idronio, $\mathrm{H}_{3} \mathrm{O}^{+}$, e l'acidità viene espressa come $\left[\mathrm{H}_{3} \mathrm{O}^{+}\right]$.

(Anche questa è una semplificazione, in quanto l'idrogeno-ione $\mathrm{H}^{+}$in una soluzione acquosa è più probabilmente legato a un "grappolo" di molecole d'acqua) [24].

È importante sapere, però, che quando la $\left[\mathrm{H}^{+}\right]$aumenta, la concentrazione "effettiva" di ioni $\mathrm{H}^{+}$liberi diventa progressivamente "minore" di quanto ci si possa attendere, a causa delle "crescenti attrazioni" tra gli ioni, le quali si generano quando le concentrazioni diventano più alte.

Questa "concentrazione effettiva" rappresenta la vera "attività" degli ioni idrogeno (o concentrazione idrogenionica "realmente efficace") ed è la misurazione considerata più corretta, anche se, di solito, il $\mathrm{pH}$ viene calcolato a partire dalla semplice relazione inversa con il logaritmo della "concentrazione" idrogenionica; bisogna, però, tener sempre conto che alle concentrazioni più elevate degli idrogenioni il pH tende a diventare meno attendibile [24]. Pertanto, è bene memorizzare che gli apparecchi di laboratorio misurano in primis le "differenze di potenziale" e le "attività", poi questi valori vengono trasformati in "concentrazioni". Gli elettrodi misuratori non rispondono alle "concentrazioni", bensì alle "attività elettriche", ed è utile sapere che la presenza di altri potenziali elettrici all'interno della soluzione (di grandezza sconosciuta) può oscurare qualsiasi misurazione. La misurazione perfetta, allora, diventa attendibile solo in condizioni ideali.

È vero che quando Sørensen per primo propose la scala del $\mathrm{pH}$, essa fu definita in termini di "concentrazione" di ioni idrogeno. Questa definizione, ormai obsoleta da tempo, continua a essere fonte di confusione.

Bisogna rendersi conto che le misure reali del $\mathrm{pH}$ con vari metodi riguardano non la "concentrazione", ma l" "attività" dello ione idrogeno ("concentrazione idrogenionica realmente efficace") [26].

In definitiva la procedura, comunemente adottata, non è concettualmente esatta, per cui "non si è legittimati a convertire sperimentalmente $i$ valori misurati di 'attività elettrica' in 'concentrazioni di ione idrogeno"' [24]; tuttavia, dal punto di vista pratico, nel sangue umano la "concentrazione" degli $\mathrm{H}^{+}$è diluita quasi all'infinito, per cui si può assumere tranquillamente la misura dell" "attività" quale misura della "concentrazione" [27], in quanto "questa distinzione teorica può essere tranquillamente ignorata nella pratica clinica" [28].

Inoltre, se una sostanza disciolta in acqua (come l'idrogeno) è dissociata, essa ha una carica, e cioè una sua attività elettrica (o ionica), ed è, quindi, più corretto parlarne in termini di equivalenza e di normalità (moli $x$ equivalenti/ litro), non di molarità (moli/litro).

Nel caso dello $\mathrm{H}^{+}$, però, che è monovalente, la normalità $\mathrm{e}$ la molarità coincidono, come pure la sua concentrazione, che nel caso della piccolissima quantità degli idrogenioni viene, oggi, comunemente espressa in forma di nanoequivalenti (valore normale $=40 \mathrm{nEq} / \mathrm{L}$, range $36-44 \mathrm{nEq} / \mathrm{L}$ ).

\section{Discussione}

Il grande capitolo della fisiologia relativo all'equilibrio acido-base è cruciale nella formazione culturale di coloro che si adoperano per il mantenimento della vita, specie nelle condizioni patologiche critiche. La didattica di questo argomento è stata per molto tempo impostata sulla famigerata equazione di Henderson-Hasselbalch ( fig. 9), in cui sono presenti "appena" (sic!) quattro entità logaritmiche: il $\mathrm{pH}$, il pK, l'acido carbonico $\left(\mathrm{H}_{2} \mathrm{CO}_{3}\right)$ e il bicarbonato $\left(\mathrm{HCO}_{3}{ }^{-}\right)$.

"Henderson, nel 1908, era stato un pioniere nel creare la sua più familiare equazione, ma sfortunatamente otto anni più tardi, nel 1916, Bjerrum, prima, e Hasselbalch, poi, rovinarono gli sforzi di Henderson, adottando i non necessari logaritmi, per produrre la terrorizzante equazione di Hasselbalch" [29].

Questa formula sostituì quella di Henderson ( fig. 9), in cui i logaritmi non comparivano e che già esprimeva benissimo lo stesso concetto in una forma alla portata di tutti i comuni mortali come noi.

Ma la ricerca della semplicità non è obiettivo di tutti.

Fu così che la formula di Hasselbalch si impose nel metodo didattico di insegnamento dell'equilibrio acido-base, mostrando a distanza di tempo, indiscutibilmente, un evidente fallimento [18].

Di chi la colpa?

La colpa è, senza alcuna ombra di dubbio, dei logaritmi negativi, capaci di confondere le idee alla maggior parte degli studenti di Medicina, i quali hanno mostrato scarsa dimestichezza con questo "artifizio matematico", finito per diventare "misterioso", "ostico" o "da evitare accuratamente", e il tutto, involontariamente, a scapito dei pazienti.

È notoria, infatti, una vera e propria avversione, anche dei medici, per questo pur importantissimo argomento, definito classicamente "ostico" ed evitato accuratamente da numerose generazioni che si trascinano tale grave lacuna culturale dalla loro formazione di base.

Lacuna che, purtroppo, finisce per incidere in maniera grave sulla vita delle persone capitate sotto la nostra gestione clinica, quando bisogna prendere decisioni terapeutiche nelle situazioni di emergenza, nei Pronto Soccorso, nelle Terapie Semintensive, nelle Rianimazioni ecc. nei casi clinici di squilibri acido-base e idroelettrolitico.

Malauguratamente, nelle attuali condizioni didattiche, per conoscere l'equilibrio acido-base bisogna conoscere il $\mathrm{pH}$ e per capire il pH bisogna aver chiaro il concetto di 
logaritmo e, come afferma Ferguson, "il concetto di pH è una delle idee più confuse in chimica e in biochimica. . . Per molti studenti [N.d.A.: quasi tutti] la necessità di dover conoscere i logaritmi è una cattiva notizia" [30].

Lo stesso Narins, uno dei massimi esperti in tema di equilibrio acido-base, nel 1980 dichiarò: "The HendersonHasselbach equation, with its reliance on logarithms and antilogarithms, is long and cumbersome and has been known to humble even the strongest of us" ("L'equazione di Henderson-Hasselbalch, con il suo riferirsi a logaritmi e antilogaritmi, è lunga e complicata ed è stata riconosciuta mortificare anche i più forti di noi”) [23].

Ovviamente, non mancarono le opinioni contrarie [31,32]: "I logaritmi negativi sono confondenti solo per coloro che non hanno voluto sopportare i modesti dolori necessari per imparare a lavorare con essi" [31], ma giustamente qualcun altro rispose: "Anche noi abbiamo imparato ad avere confidenza con la scala del $\mathrm{pH}$, ma siamo convinti che l'uso della $\left[\mathrm{H}^{+}\right]$sia più chiaro e molto più facile da insegnare" [31].

In fondo, però, se ci si avvicina al tema con buona volontà, senza soggezione, con umiltà e senza prevenzioni (senza, cioè, freni inibitori precostituiti), si può scoprire che esso è semplice da assimilare e da far proprio, senza grande dispendio di energie.

Basti vedere la semplicità dell'equazione di Henderson quando nella formula generale si introducono i valori dei parametri normali dell'uomo in fase di equilibrio fisiologico ( fig. 16). Il valore numerico della concentrazione degli idrogenioni è dato dal numero 24 (la costante) moltiplicato x 40 (la pressione normale della $\mathrm{CO}_{2}$ ), diviso per 24 (il valore normale dei bicarbonato). I due numeri 24 si elidono e ne risulta: $\left[\mathrm{H}^{+}\right]=40$ ( fig. 17 ).

Con questo tipo di formula i calcoli sono semplicissimi, alla portata dei ragazzi delle scuole elementari, in quanto si fa ricorso solo a semplici moltiplicazioni e divisioni. Tutto ciò diventa cruciale, per esempio, quando si debbano calcolare le quantità di bicarbonati da infondere in un paziente con acidosi metabolica oppure quando bisogna calcolare i compensi attesi.

Proviamo a immaginare, invece, le difficoltà a dover fare tali calcoli con l'equazione di Hasselbalch e con i logaritmi.

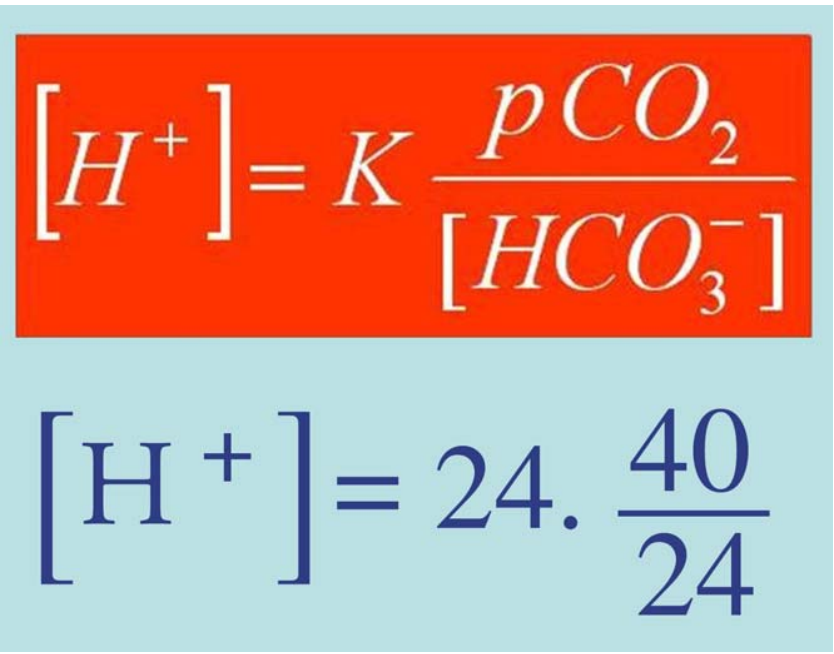

Figura 16 Valori numerici dell'equazione di HendersonCampbell.

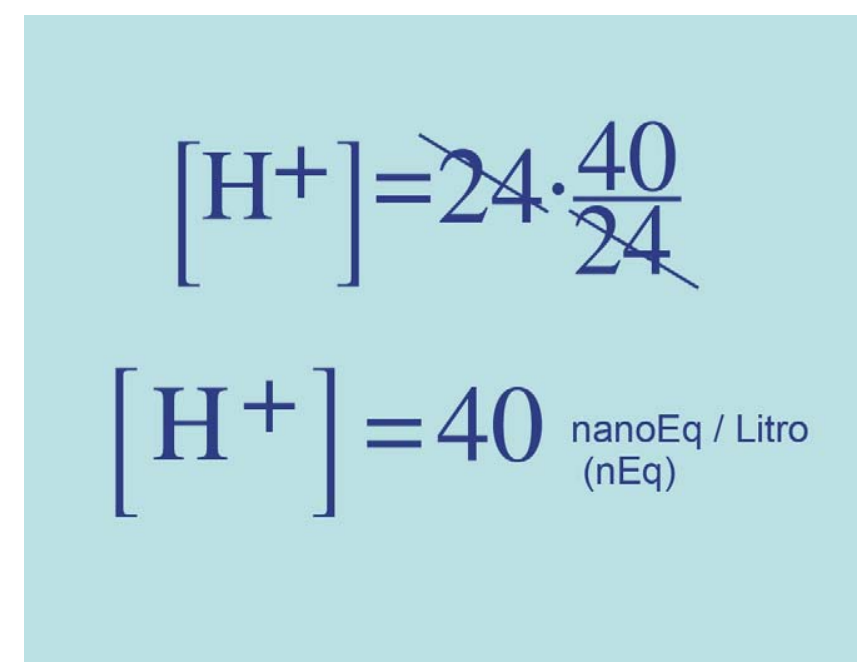

Figura 17 Risoluzione dell'equazione in chiave numerica.

\section{Conclusioni}

Non si ha l'ardire di pensare di poter eliminare il pH dal mondo scientifico, in particolare da quello chimico e biochimico, ma sicuramente bisognerà battersi, finché ne avremo la forza, per farlo scomparire dalla didattica iniziale dell'equilibrio acido-base in Medicina.

Il dibattito su questo delicato argomento non è nato adesso, ma procede da almeno cinquant'anni e ultimamente si sta risolvendo a favore dei nanoequivalenti contro i logaritmi, cioè a favore di Henderson contro Hasselbalch (finalmente!) $[1,13-15,22,23,26,28,29,31,33-50]$.

Da quanto detto appare chiaro che la presenza del pH e dei logaritmi non aggiunge nulla alla comprensione di questa cruciale tematica della fisiologia e della patologia generale e, anzi, è dannosa per la classe medica (giovane e non) e per tutti i pazienti che si affidano alle nostre cure.

Il pH (logaritmo negativo in base 10 della concentrazione idrogenionica) e l'equazione di Henderson-Hasselbalch sono praticamente "superflui" ai fini della comprensione nella didattica in Medicina e possono essere sostituiti, con estrema semplicità, rispettivamente, dalla concentrazione idrogenionica (in sigla $\left[\mathrm{H}^{+}\right]$) e dall'originaria equazione di Henderson.

L'auspicio è che chiunque abbia responsabilità didattiche (e tenga a cuore l'amore per il trasferimento della cultura alle nuove generazioni di medici e, quindi, la vita dei propri pazienti) si impegni a escludere gli "artifizi matematici" e le "formule ingombranti", utili solo a complicare l'approccio al tema e a suscitare la nota avversione che, poi, gli studenti trascinano con sé per tutta la loro vita professionale.

Avversione che porta a escludere dalle proprie nozioni di base un capitolo molto importante, tra l'altro vitale in molte condizioni cliniche, e che, una volta appreso con semplicità, senza gli odiosi e superflui logaritmi negativi, diventa uno degli argomenti più affascinanti e amabili della fisiopatologia generale.

Non si può, allora, non condividere l'appello lanciato da Seeman [47]: "Unisciti alla crociata per sostituire l'insegnamento del $\mathrm{pH}$ con l'insegnamento della concentrazione di 


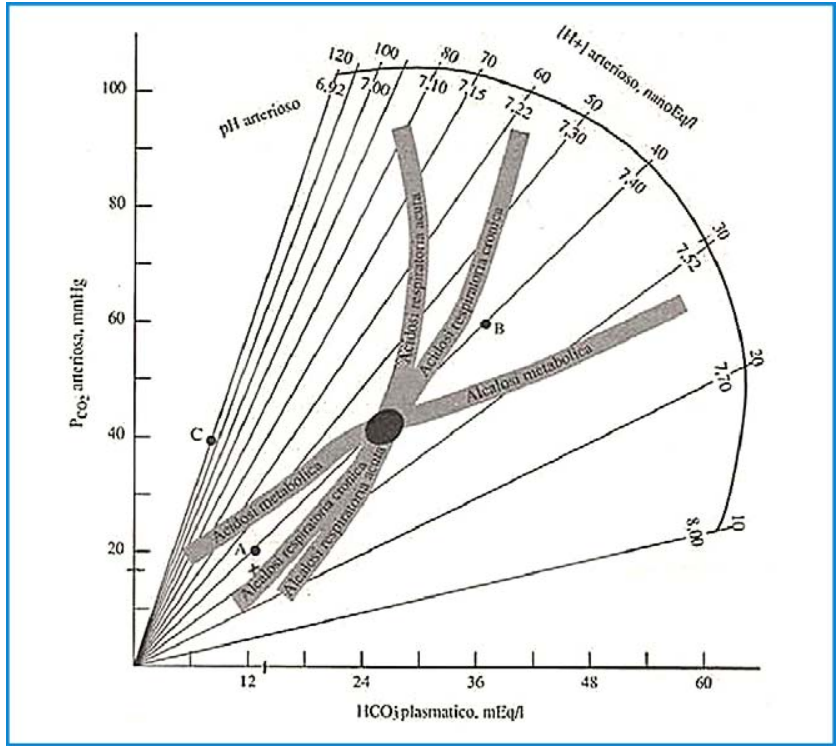

Figura 18 Nomogramma con la presenza anche degli idrogenioni espressi in nanoequivalenti per litro.

protoni in tutti i corsi di laurea. Abbiamo bisogno di un'organizzazione per promuovere questa causa" [N.d.A.: che anche noi consideriamo, senza alcun dubbio, benefica].

L'avvio allo studio può e deve cominciare con la sola equazione di Henderson (senza logaritmi).

Non è indispensabile conoscere l'equazione di Hasselbalch.

Altra conquista da ottenere subito, in ambito industriale, è che le aziende produttrici di emogasanalizzatori impostino il software degli apparecchi in modo da aggiungere ai referti anche il valore degli idrogenioni $\left[\mathrm{H}^{+}\right]$(espressi in nanoequivalenti) prima del valore del $\mathrm{pH}$, com'è stato realizzato in molti nomogrammi pratici che hanno cominciato ad adeguarsi [51] ( fig. 18).

In definitiva, dobbiamo convincerci che nell'equilibrio acido-base gli elementi veramente importanti sono solo tre: gli idrogenioni, la $\mathrm{pCO}_{2}$ e i bicarbonati. Il resto è tutto una sovrastruttura che qualcuno ha messo in piedi, in buona fede, senza accorgersi che complicava la vita alla gente.

Questo nuovo modo di studiare l'equilibrio acido-base, collegato all'equilibrio idroelettrolitico e osmolare, rende l'approccio meno ostico [52,53] e ci permette di meglio capire che, nella nostra attività lavorativa quotidiana, nulla di quanto facciamo routinariamente non include o non interferisce su tali materie. Tutte considerazioni che fanno di questo tema un argomento di cui dobbiamo occuparci per forza. L'equilibrio acido-base, infatti, è una di quelle aree della medicina che non può essere considerata circoscritta a un settore organo-specifico, bensì è trasversale a tutte le specialità mediche, fungendo da argomento unificante insieme a quello idro-elettrolitico [52,54].

Nessun medico operante al letto del paziente può ritenersi non interessato a questi argomenti, in special modo, poi, nei settori dell'emergenza, della medicina interna e delle terapie intensive e semi-intensive. Ovviamente, la problematica acido-base è ancora più complessa e coinvolge le interrelazioni ineludibili con gli altri equilibri idro-elettrolitico, osmolare, volemico, ormonale, cardio-circolatorio, etc..

Gli attori in campo non sono solo l'idrogeno, l'ossigeno e il sistema acido carbonico-bicarbonato, ma contano anche gli altri sistemi tampone (proteine, fosfati, emoglobina), gli ioni forti (sodio, cloro, potassio, etc...), gli acidi deboli, etc. come si può evincere da lavori più moderni (Ipotesi di Stewart) [52,55-59].

Ma come si può parlare di questa ulteriore complessità, agli studenti e ai medici meno motivati, se essi non hanno ancora digerito $i$ concetti fondamentali a causa dei logaritmi di Hasselbalch?

Per tali motivi anche gli altri importanti e utili concetti di "gap anionico" o di "strong ions difference" vengono visti con diffidenza e, verso di loro, scatta la stessa avversione, fino alla vera e propria reazione di rigetto.

Al contrario, poi, è esperienza comune che, una volta che il tema sia stato piacevolmente appreso senza i logaritmi, successivamente diventa facile capirlo anche con l'equazione di Henderson-Hasselbalch (con i logaritmi).

A questo punto, chi ama ancora utilizzare i logaritmi può sempre continuare a farlo, perpetuando il suo masochismo biochimico-matematico, ma, almeno, eviti alle nuove generazioni il suo involontario sadismo didattico in Medicina.

\section{Conflitto di interesse}

Gli autori dichiarano di essere esenti da conflitto di interessi.

\section{Bibliografia}

[1] Elkinton JR. Hydrogen-ionized and otherwise. Ann Int Med 1962;57(4):687-8.

[2] Kraut JA, Coburn JW. Bone, acid, and osteoporosis. N Engl J Med 1994;330(25):1821-2.

[3] Bernard C. Introduction à l'étude de la médecine expérimentale. Paris: JB Baillere et Fils, 1865. Tr. it. Introduzione allo studio della medicina sperimentale. Padova: Piccin; 1994.

[4] Henderson LJ. Concerning the relations between the strenght of acids and their capacity to preserve neutrality. Am J Physiol 1908;21:173-9.

[5] Henderson LJ. The theory of neutrality regulation in the animal organism. Am J Phisiol 1908;21:427-48.

[6] Lowenstein J. Henderson's Equation. Egremont, MA: Gadd \& Company; 2008.

[7] Loeb J. The organism as a whole: from a physicochemical viewpoint. New York (NY), London: GB Putnam's Sons; 1916.

[8] Loeb J. The origin of the conception of physiologically balanced salt solutions. J Biol Chem 1918;34(3):503-4.

[9] Sgambato F, Sgambato E, Fucci A. La formula di Loeb: una ricca eredità dissipata. Emerg Care J 2006;II(IV):13-20.

[10] Cannon WB. The Wisdom of the Body. New York, NY: Norton; 1932 (rist. London: Peter Smith, 1979).

[11] Sørensen SPL. Enzymstudien. II. Uber die Messung und die Bedeutung der Wasserstoffionenkonzentration bei Enzymatischen Prozessen. Biochem Z 1909;21(131):201.

[12] Vulterini S. Le basi fisiopatologiche dell'equilibrio acido-base. II Policlinico Sezione Pratica 1994;101(21):720-52.

[13] Sgambato F, Prozzo S, Milano L, Sgambato E, Piscitelli GL, Fucci A. Gli equilibri omeostatici in Medicina: dalla fisiopatologia alla filosofia. Conferenza all'Accademia Lancisiana. Il Policlinico Sezione Pratica 2009;116:I146-66. 
[14] Sgambato F. Un nuovo teorema. Ovverosia può la filosofia napoletana essere applicata all'equilibrio acido-base?. Ah saperlo, saperlo. Eur Resp News 1995;3(2):45-8.

[15] Campbell EJ. RIpH. Lancet 1962;1(7231):681-3.

[16] De Crescenzo L. Panta rei (tutto scorre). Milano: Mondadori; 1994.

[17] Hasselbalch KA. Die Berechnung der Wasserstoffzahl der Blutes aus der frien gebundenen Kahlensaure desselben, und die Sauerstoffbindung des Blutes als Funktion der Wasserstoffzahl. Biochem Z 1916;78:112.

[18] Po HN, Senozan NM. The Henderson-Hasselbalch equation: Its history and limitations. J Chem Educ 2001;78(11):1499-503.

[19] de Levie R. The Henderson-Hasselbalch equation: Its history and limitations. J Chem Educ 2003;80:146.

[20] Guldberg CM, Waage P. Studies concerning affinity. Forhandl. Videnskabs-Selskabe Christiania 1865;35-45.

[21] Guldberg CM, Waage P. Concerning chemical affinity. Erdmann's J Practische Chemie 1879;19(2):69-114.

[22] Schwartz WB, Relman AS. A critique of the parameters used in the evaluation of acid-base disorders. "Whole-blood buffer base" and "standard bicarbonate" compared with blood $\mathrm{pH}$ and plasma bicarbonate concentration. N Engl J Med 1963;268:1382-8.

[23] Narins RG, Emmett M. Simple and mixed acid-base disorders: a practical approach. Medicine (Baltimore) 1980;59(3):161-87.

[24] Kolb D. The pH concept. J Chem Education 1979;56:49-53.

[25] Watters DJ, Watters JJ. Student understanding of pH: "I don't know what the log actually is I only know where the button is on my calculator". Biochem Mol Biol Educ 2006;34(4):278-84.

[26] Butler TC. pH: another view. Science 1973;179(4076):854-5.

[27] Polli E, Fieschi A. Le alterazioni dell'equilibrio acido-base. Atti del LXXIV Congresso della Società Italiana di Medicina Interna (SIMI). Montecatini, 1973.

[28] Flenley DC. Another non-logarithmic acid-base diagram? Lancet 1971;1(7706):961-5.

[29] Grogono AW. Acid-Base Tutorial. www.acid-base.com/terminology.php.

[30] Ferguson JB. Exercise 4: Titration of an amino acid. http:// biology.bard.edu/ferguson/course.

[31] Hills AG, Reid EL, Lennon EJ, Lemann Jr JL. pH defended-Is it defensible? Ann Intern Med 1966;65(5):1150-2.

[32] Davis RP. Logland: a Gibbsian view of acid-base balance. Am J Med 1967;42(2):159-62.

[33] Lennon EJ, Lemann Jr J. pH defended - is pH defensible? (Reply) Ann Inter Med 1966;65(5):1151-2.

[34] Myers RJ. One-hundred years of pH. J Chem Educ 2010;87(1): $30-2$.

[35] Sgambato F. L'equilibrio acido-base nella pratica clinica senza logaritmi. Relazione presentata al III Congresso Nazionale FADOI maggio 1998. Internista Ospedaliero 1999;3-4(3/98-1/99):21-7.

[36] Sgambato F, Prozzo S, Pedicini A, Caporaso C. The acid base balance in clinical practice without logarithms. In: De Santo NG (ed). Acid-base balance. From bench to bedside. Atti del
Congresso Mondiale dell'Istituto Italiano di Studi Filosofici di Napoli (Anacapri, ottobre 1999), pp. 307-13.

[37] Crane Jr FE. Is there an alternative to $\mathrm{pH}$ ? J Chem Educ 1961;38:365-6.

[38] Huckabee WE. Henderson versus Hasslebalch. Clin Res 1961; 9:116-9.

[39] Szabadváry F, Oesper RE. Development of the pH concept. A historical survey. J Chem Educ 1964;41(2):105-7.

[40] Owen JA, Dudley HA, Masterton JP. Acid-base status assessed from measurements of hydrogenion concentration and PCO2. Lancet 1965;2(7414):660-2.

[41] Lennon EJ, Lemann Jr J. Defense of hydrogen ion concentration in chronic metabolic acidosis. A new evaluation of an old approach. Ann Intern Med 1966;65(2):265-74.

[42] Hills AG, Reid EL. More on pH. Ann Intern Med 1967;66(1):238-9.

[43] Hills AG. pH and the Henderson-Hasselbalch equation. Am J Med 1973;55(2):131-3.

[44] Howorth PJ. RIpH revisited. Lancet 1974;1(7851):253-4.

[45] Fulop M, Fulop M. Acid-base diagrams maths, myths, and measurements. Lancet 1974;2(7881):637-9.

[46] Neiberger RE. Removing the pH from pHysician pHrases. JAMA 1991;266(18):2559.

[47] Jensen WB. The symbol for pH. J Chem Educ 2004;81(1):21.

[48] Seeman P. The pH concept. Science 1972;177(4052):835-6.

[49] Kassirer JP, Bleich HL. Rapid estimation of plasma carbon dioxide tension from $\mathrm{pH}$ and total carbon dioxide content. $\mathrm{N}$ Engl J Med 1965;272:1067-8.

[50] Astrup P, Severinghaus JW. The History of Blood Gases, Acids and Bases. Copenhagen: Radiometer A/S; 1986.

[51] Rose DB. Fisiologia clinica dell'equilibrio acido-base e dei disordini elettrolitici. Milano: McGraw-Hill; 1995.

[52] Sgambato F. Gli equilibri omeostatici in Medicina: le interrelazioni fra i sistemi. Atti del X Congresso Nazionale FADOI. GIMI 2005;4(1 Suppl 2):13-23.

[53] Sgambato F, Prozzo S, Sgambato E, Sgambato R, Milano L. The 100th anniversary of $\mathrm{pH}$ (1909-2009). Parte prima. Negative logarithms for measuring hydrogenions: Are they essential in medicine? Ital J Med 2011;5:147-55.

[54] Kellum JA. Determinants of blood pH in health and disease. Crit Care 2000;4:6-14.

[55] Stewart PA. How to understand acid-base. In: Stewart PA, editor. A Quantitative Acid-Base Primer for Biology and Medicine. New York: Elsevier; 1981. p. 1-286.

[56] Stewart PA. Modern quantitative acid-base chemistry. Can J Physiol Pharmacol 1983;61:1444-61.

[57] Severinghaus JW. Sigaard-andersen and the Great Trans Atlantic Acid-Base Debate. Scand J Clin Lab Invest 1993;53(Suppl 214): 99-104.

[58] Corey HE. Stewart and beyond: New models of acid-base balance. Kidn Intern 2003;64:777-87.

[59] Kurtz I, Kraut J, Ornekian V, Nguyen MK. Acid-Base: A Critique of the Stewart and Bicarbonat Centered Approaches. Am J Physiol Renal Physiol 2008;294:F1009-31. 